\title{
Predicting color from gray: the relationship between achromatic adjustment and asymmetric matching
}

\author{
Jon M. Speigle* and David H. Brainard \\ Department of Psychology, University of California, Santa Barbara, Santa Barbara, California 93106
}

Received December 21, 1998; revised manuscript received May 7, 1999; accepted May 20, 1999

\begin{abstract}
Achromatic adjustment has been used widely to study color context effects. In the achromatic adjustment procedure, an observer adjusts a test stimulus until it appears black, gray, or white. By its nature, achromatic adjustment directly measures the effect of context only for stimuli that appear gray. We present achromatic loci measured in two contexts and asymmetric color matches measured across the same two contexts. The results indicate that achromatic adjustments, together with a gain-control model, may be used to make accurate predictions of the chromaticity of asymmetric matches. Thus measurements of the effect of context for test stimuli that appear gray may be used to predict the effect of context for stimuli that appear colored. The experiments also indicate that accurate prediction depends on ensuring that observers use similar fixational strategies for the two judgments. (c) 1999 Optical Society of America [S0740-3232(99)02909-9] OCIS codes: $330.1690,330.1720,330.1710$.
\end{abstract}

\section{INTRODUCTION}

Achromatic adjustment has been used widely to study chromatic adaptation and other color context effects. ${ }^{1-9}$ In the achromatic adjustment procedure, an observer adjusts a test stimulus until it appears black, gray, or white. Typically, the observer is allowed to vary the chromaticity of the stimulus while its luminance is held constant.

The achromatic adjustment procedure has several advantages. First, the judgment is easy for naive observers, and observers' settings tend to be reliable. Second, because the judgment requires no external matching standard, it is straightforward to employ in experiments where adaptation is carefully controlled. Finally, the data are powerful enough to support quantitative models of color context effects.

On the other hand, achromatic adjustment measures directly the effect of context only for stimuli that appear gray. This fact may limit the usefulness of the procedure for understanding how color context affects colored stimuli.

Here we test whether measured achromatic loci permit accurate prediction of the effect of context on stimuli that are not achromatic. We measured achromatic adjustments in two contexts and asymmetric matches across the same two contexts. We used the same observers for the two types of measurement and modeled both data sets by using the same class of simple gain-control model. Within the context of this model we assessed how well each observer's achromatic adjustments could be used to predict his or her asymmetric matches.

For achromatic adjustments to allow accurate prediction of asymmetric matches, several conditions must hold. First, both types of judgment must be affected by context in a consistent fashion. For example, if a contextual manipulation affects one of the judgments but not the other, then accurate prediction will not be possible. Second, there must be a model that links the two types of judgment. The parameters of such a model should be determined by the achromatic adjustments, and once these parameters are determined, the model should predict properties of the asymmetric matches. Finally, as a practical consideration, the precision of the achromatic adjustments must be high enough that the model-based predictions are not distorted by measurement error.

In this paper we examine the relationship between achromatic adjustment and asymmetric matching for scenes consisting of illuminated objects where the context is manipulated by changing the illuminant. This type of contextual manipulation is of particular interest since it is commonly employed in studies of color constancy. ${ }^{5-7,9-17}$

\section{METHODS}

A. Asymmetric Matching

The asymmetric matching data have been reported previously. ${ }^{17}$ Observers adjusted a match surface until it appeared as similar as possible to a test surface and then reported the quality of the match ("satisfactory" or "unsatisfactory"). The test and match surfaces were presented on the left and right of the far wall of an experimental room (see Ref. 17, Fig. 1). The room lighting was arranged so that there was an illumination gradient from left to right. The light at the test location was bluish, and the light at the match location was yellowish. The chromaticities and luminances of the illuminants at the test and match locations differed slightly from observer to observer and are given in Table 1.

The light reflected from the match surface to the observer consisted of two components. The first was from the ambient illumination, and the second was generated 
by a computer-controlled projection colorimeter. This second component was spatially coincident with the match surface. Observers adjusted the appearance of the match surface by varying the output of the colorimeter.

The illumination gradient changed the context in which the test and match surfaces were seen, and as a result there was a difference between the tristimulus coordinates of the test and match surfaces when they appeared identical to the observer. This difference is a measure of the effect of the bluish-to-yellowish illumination gradient on color appearance. $15,17,18$

Each observer made 2-4 appearance matches to approximately 30 test surfaces by adjusting the output of the projection colorimeter. When the observer achieved a satisfactory match, we placed a spectrophotometer (Photo Research PR-650) at the position of the observer and measured the tristimulus coordinates of the test and match surfaces. When a match was unsatisfactory, we shifted the device gamut by changing the surface at the match position, and the observer attempted the matching process again. We report only the data corresponding to satisfactory matches.

\section{B. Achromatic Adjustment}

The achromatic adjustments were made in the same experimental room as the asymmetric matches, and observers again adjusted the match surface. The general procedures were similar to those reported in Ref. 9. In the achromatic adjustment experiments, observers adjusted the chromaticity of the match surface while its luminance was held approximately constant. For most of our conditions, this procedure was repeated at several different match surface luminances.

Achromatic adjustments were made under two primary illumination conditions. One was identical to that used in the asymmetric matching experiments. We refer to this as the yellowish illumination condition. The other illumination condition was obtained by a left-to-right reversal of the illuminants in the room. In this condition, the illuminant at the match surface was bluish, the same as it was at the test surface in the asymmetric matching experiment. In addition, there was an illumination gra- dient across the front of the room, so that the illuminant changed to yellowish at the left side of the room. We refer to this as the bluish illumination condition. The chromaticities and luminances of the illumination at the test and match locations for each observer are given in Table 1. (In the achromatic adjustment experiment, the test surface was not relevant to the experimental task, but we provide the illuminant at its location for comparison with the asymmetric matching experiment).

At the beginning of each experimental session, the observer adapted to the illumination condition for $5 \mathrm{~min}$. The observer then adjusted the match surface until it appeared achromatic (i.e., black, gray, or white). At the start of the adjustment, the CIELAB $a *$ and $b^{*}$ chromaticity was set to a random value in the range $-25 \leqslant a^{*}$ $\leqslant 25,-25 \leqslant b^{*} \leqslant 25$, with respect to a white point defined by the illuminant at the match surface. This is the basic starting rule described in Ref. 9. The observer adjusted chromaticity by using two knobs that controlled the $a^{*}$ and $b *$ coordinates of the light reflected from the match surface. The luminance of the match surface was fixed throughout an adjustment. The observer terminated a trial by pressing one of two buttons to indicate either a "satisfactory" or an "unsatisfactory" adjustment. Unsatisfactory terminations resulted because at some luminance levels the stimulus gamut did not contain an achromatic point. Only satisfactory adjustments were used in the data analysis.

At least four achromatic adjustments were made by each observer in each illumination condition. Stimulus luminances varied across conditions. Table 2 presents the number of adjustments and stimulus luminances for each observer/condition pair. The majority of the luminance levels produced stimuli that appeared as surfaces, although at the highest luminance levels some may have appeared self-luminous. ${ }^{19}$ As described in Ref. 17 for the asymmetric matches, different physical surfaces were used at the match location to manipulate the stimulus gamut from session to session. In the achromatic adjustment experiments, the physical surface was always a matte, neutral Munsell paper: N2/, N3/, N3.5/, or N5/. A Munsell N3/ paper was placed at the test surface location.

Table 1. Illuminant Specifications: Measured CIE 1931 x y Chromaticity and Luminance $\left(\mathrm{cd} / \mathrm{m}^{2}\right)$ of the Illuminant at Test and Match Locations for Each Observer, Experiment, and Illuminant ${ }^{\mathrm{a}}$

\begin{tabular}{|c|c|c|c|c|c|c|c|c|}
\hline Illuminant & Experiment & Observer & Test $\mathrm{x}$ & Test y & $\begin{array}{c}\text { Test } \\
\text { Luminance }\end{array}$ & Match $\mathrm{x}$ & Match y & $\begin{array}{c}\text { Match } \\
\text { Luminance }\end{array}$ \\
\hline Yellowish & ASM & $\mathrm{ASH}, \mathrm{J} M S$ & 0.349 & 0.356 & 19.8 & 0.451 & 0.399 & 83.8 \\
\hline Bluish & $\mathrm{ACH} \mathrm{A}$ & $\mathrm{ASH}, \mathrm{J} M S$ & 0.455 & 0.399 & 80.1 & 0.351 & 0.349 & 19.0 \\
\hline Yellowish & $\mathrm{ACH} \mathrm{A}$ & $\mathrm{ASH}, \mathrm{J} M S$ & 0.347 & 0.351 & 19.5 & 0.452 & 0.397 & 84.4 \\
\hline Bluish & $\mathrm{ACH} \mathrm{S}$ & $\mathrm{ASH}, \mathrm{J} M S$ & 0.450 & 0.400 & 81.1 & 0.344 & 0.357 & 19.7 \\
\hline Yellowish & $\mathrm{ACH} \mathrm{S}$ & $\mathrm{ASH}, \mathrm{J} M S$ & 0.346 & 0.353 & 20.8 & 0.449 & 0.397 & 84.8 \\
\hline Yellowish & ASM & PBE & 0.371 & 0.353 & 16.5 & 0.456 & 0.398 & 72.0 \\
\hline Bluish & $\mathrm{ACH} A$ & PBE & 0.460 & 0.397 & 70.4 & 0.379 & 0.346 & 15.5 \\
\hline Yellowish & $\mathrm{ACH} \mathrm{A}$ & PBE & 0.372 & 0.346 & 15.9 & 0.456 & 0.395 & 71.9 \\
\hline Bluish & $\mathrm{ACH} \mathrm{S}$ & PBE & 0.456 & 0.397 & 71.3 & 0.373 & 0.351 & 16.5 \\
\hline Yellowish & $\mathrm{ACH} \mathrm{S}$ & PBE & 0.372 & 0.357 & 16.9 & 0.457 & 0.399 & 73.4 \\
\hline
\end{tabular}

${ }^{\text {a ASM }}$, asymmetric matching; ACH A, achromatic adjustment, alternating fixation; ACH S, achromatic adjustment, steady fixation. 
Table 2. Achromatic Adjustment Conditions and Results: Number of Adjustments Made, Mean CIE 1931 xy Chromaticity of the Adjustments, and Luminances $\left(\mathrm{cd} / \mathrm{m}^{2}\right)$ at Which Adjustments Were Made ${ }^{a}$

\begin{tabular}{lcccccc}
\hline Illuminant & Fixation & Observer & Number & Mean x & Mean y & Luminances \\
\hline Bluish & A & ASH & 4 & 0.351 & 0.365 & 3 \\
Yellowish & A & ASH & 8 & 0.425 & 0.395 & 6,10 \\
Bluish & S & ASH & 4 & 0.333 & 0.376 & 3 \\
Yellowish & S & ASH & 84 & 0.430 & 0.397 & $3-40$ \\
Bluish & A & J MS & 12 & 0.363 & 0.372 & $3,6,10$ \\
Yellowish & A & J MS & 16 & 0.434 & 0.398 & $4,6,8,10$ \\
Bluish & S & J MS & 8 & 0.337 & 0.364 & 3,6 \\
Yellowish & S & J MS & 112 & 0.439 & 0.400 & $3-40$ \\
Bluish & A & PBE & 8 & 0.371 & 0.361 & 4,8 \\
Yellowish & P & PBE & 16 & 0.424 & 0.392 & $6,10,14,18$ \\
Bluish & S & PBE & 8 & 0.363 & 0.367 & 4,8 \\
Yellowish & S & PBE & 68 & 0.431 & 0.402 & $3-20$ \\
\hline
\end{tabular}

a When the luminances are specified by a range, adjustments were made at a large number of luminances within the range. A, alternating fixation; S, steady fixation.

In an asymmetric matching experiment, it is natural for the observer to look back and forth between the test location and the match location as he or she compares the appearance of the test and the match surfaces. In an achromatic adjustment experiment, observers need only compare the match surface to an internal standardother locations are irrelevant. Thus in an achromatic adjustment experiment it is natural for the observer to fixate primarily on the match surface. Because we wanted the stimulus conditions in the asymmetric matching and the achromatic adjustment experiments to correspond as closely as possible, we instructed our observers to look back and forth between the match and test locations in the achromatic adjustment experiment every $5 \mathrm{~s}$. Compliance with these instructions was facilitated by use of a speech synthesizer, which played the words "left" and "right" to the observer at the appropriate intervals. We refer to this as the alternating fixation condition. For comparison, we also ran a steady fixation condition where the observers were not instructed to vary their fixation. In this condition, observers tended to fixate primarily on the match surface.

\section{Observers}

Three observers participated in the asymmetric matching experiments. Observer ASH, age 27, female, was paid for her participation. Observer PBE, age 32, male, was a graduate student volunteer. Observer JMS, age 29, male, was the first author. All observers had normal color vision as determined with the Ishihara pseudoisochromatic plates.

\section{Calibration}

The calibration procedures for illumination and projection colorimeter control are described in Ref. 17. Of note is that the asymmetric and the achromatic adjustment data reported were obtained by replaying observers' adjustments and making direct spectral measurements of the light reflected to the observer from the match surface (Photo Research PR-650). Direct measurements of the il- luminant at the test and match locations were also made in each session (Photo Research RS-2 reference, 99.9\% reflectance).

\section{RESULTS}

A. Asymmetric Matching

Figure 1 shows typical data for the asymmetric matching experiment. The panels on the left plot CIE y chromaticity against $\mathrm{CIE} \times$ chromaticity, and the right panels plot luminance against $\mathrm{CIE} \times$ chromaticity. Each open square shows the coordinates of one test surface, and the connected solid square shows the coordinates of the corresponding match surface. The difference between test and match coordinates measures the effect of the illuminant gradient on color appearance. If there were no effect of the illuminant gradient, the open and solid squares would superimpose. The data indicate that the illumination gradient had a substantial effect, for both chromaticity and luminance. Data for the other two observers are similar. The entire asymmetric matching data set is provided in Table 2 of Ref. 17.

The asymmetric matching experiment establishes correspondence between stimuli that appear identical across the change in context. To the extent that identity of appearance is independent of the experimental method used to assess it, asymmetric matches made for a particular change of context provide the empirical basis for a complete characterization of the effect of that change on color appearance. For many context changes, asymmetric matches are well described by a simple gain-control model. ${ }^{15,17,20,21}$ In this type of model, the only effect of context is to change the gains on signals from the three [long-, middle, and short-wavelength-sensitive ( $L, M$, and $S$, respectively)] classes of cone photoreceptors. This type of gain control is often referred to as von Kries adaptation 22 ; here we refer to the model as the diagonal model. ${ }^{15,17}$ When the diagonal model holds, the cone coordinates of the match surfaces are related to the cone coordinates of the test surfaces by a diagonal linear transformation. Although there are conditions under which 
the diagonal model fails to describe the effects of changes of context, ${ }^{23-25}$ we found that it fit our matching data nearly as well as models in which the transformation was a fully populated $3 \times 3$ matrix or where the transformation included additive terms. ${ }^{17}$
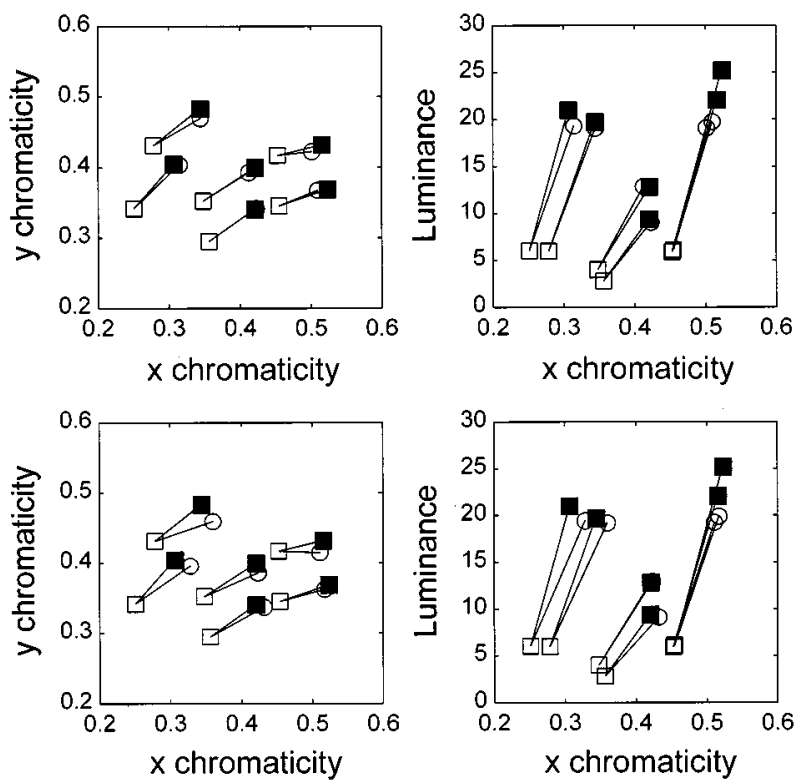

Fig. 1. Asymmetric matches. Top panels show data and predictions for the diagonal model: open squares, test surface coordinates; solid squares, match surface coordinates; open circles, matches predicted by the diagonal model. Bottom panels are the same as the top ones except that model predictions are constrained by the achromatic loci measured with alternating fixation. Correspondence between test and match coordinates is indicated by the solid lines. Data are for observer ASH. The standard error of measurement is smaller than the plotted points. To avoid overwhelming the plots, only a subset of the data is shown. Similar data for observer J MS are shown in Ref. 17, Fig. 6.
The top panels of Fig. 1 illustrate how well the diagonal model fits the asymmetric matching data from our experiment. The open circles show the diagonal model predictions, and the solid squares indicate the observer's matches. It is clear that the predictions generally lie near the matches. Details of the fitting procedure and an analysis of the quality of fit are provided in Ref. 17.

\section{B. Achromatic Adjustment}

Achromatic adjustments were made at a number of match surface luminances (see Section 2). Previous authors have found that when the adjusted stimulus is an incremental uniform field seen on a uniform background, the chromaticity that appears achromatic varies with luminance. ${ }^{1,3,8}$ For conditions similar to ours, however, no such dependence is found; the chromaticity that appears achromatic is independent of match surface luminance over the luminance range that we used., 9,26 Thus for our conditions it is possible to describe the achromatic locus by a single chromaticity. We computed this chromaticity as the mean (over luminance and replication) of the chromaticities of the individual achromatic adjustments.

Figure 2 shows the CIE xy chromaticities of the achromatic loci for the three observers. The top panels show the data for the alternating-fixation condition, and the bottom panels show the data for the steady-fixation condition. The open square shows the achromatic locus when the illumination at the match surface was bluish (bluish illuminant), and the solid square shows the locus when the illumination at the match surface was yellowish (yellowish illuminant). The chromaticities of the corresponding illuminants are shown by the open and solid triangles. The achromatic point shifts systematically with the change in illuminant, and the size and direction of the
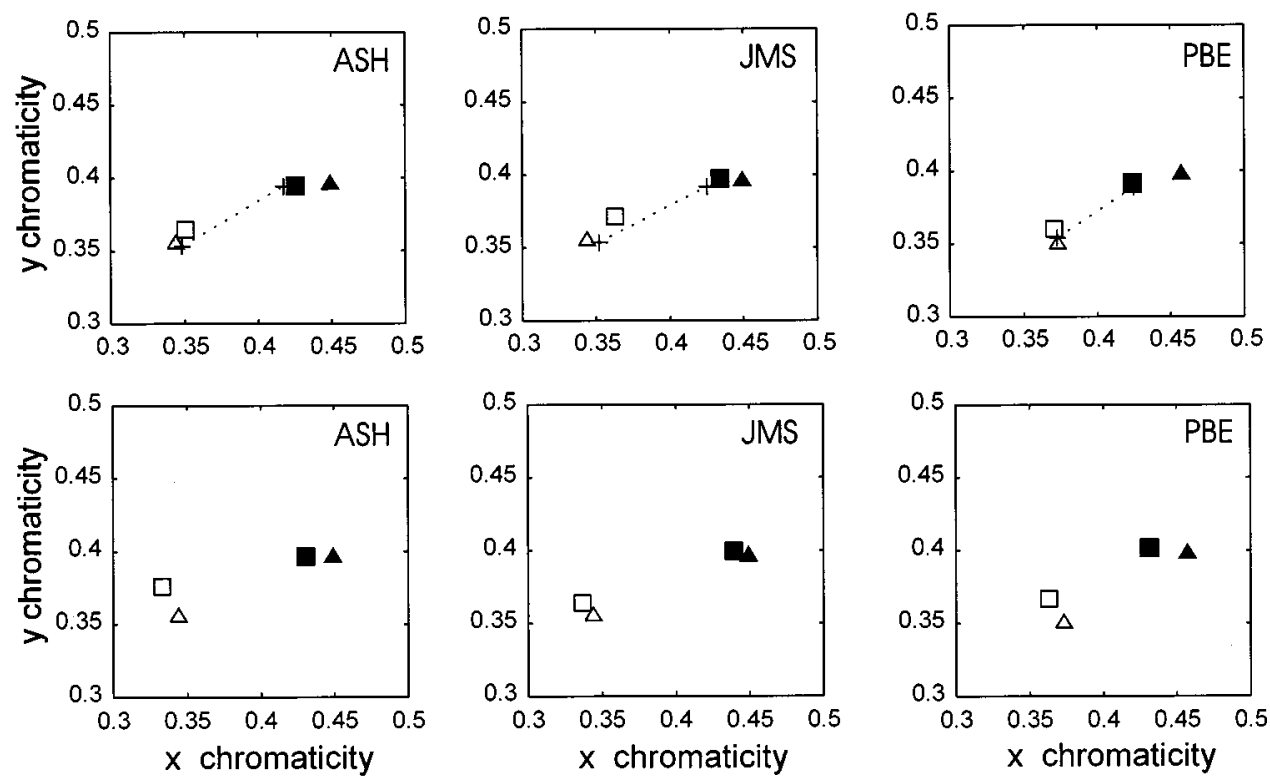

Fig. 2. Achromatic loci. Top panels show the alternating-fixation condition. Bottom panels show the steady-fixation condition. The $\mathrm{CIE}$ xy chromaticities of the achromatic loci for the three observers are shown: open squares show the achromatic locus when the illumination at the match surface was bluish (bluish illuminant); solid squares show the locus when the illumination at the match surface was yellowish (yellowish illuminant); open and solid triangles show the corresponding illuminants. In the top panels, the dashed lines show asymmetric matching data for comparison (see description in text). 
shift is similar to that seen for the asymmetric matches, particularly for the data from the alternating-fixation condition.

\section{Consistency of Matching and Achromatic} Adjustment

If the achromatic adjustments are consistent with the asymmetric matches, then the chromaticity shift that is revealed in the asymmetric matches for test surfaces that appeared achromatic should be similar to the shift seen in the achromatic loci for the alternating-fixation condition. For each observer we found test surfaces whose chromaticities were close to the achromatic locus measured under the bluish illuminant. The asymmetric matching data for these test surfaces is shown as dotted lines in the top panels of Fig. 2. The ends of each line represent the test and match surface chromaticities for a single test surface. Note that the dotted lines are in good agreement with the achromatic loci. This indicates that the two types of measurements are affected similarly by the change in illuminant and suggests that they tap the same underlying perceptual representation. As noted in Section 1 , this is a necessary condition for using achromatic adjustments to predict asymmetric matches.

D. Prediction of Matching from Achromatic Adjustment Predicting the asymmetric matches from the achromatic adjustments requires a model. Since the diagonal model provides a good description of the asymmetric matching data, we use it for this purpose. The diagonal model has three parameters, one for each cone class. In principle, the parameters may be determined from a single asymmetric match. The achromatic adjustments establish two loci, each of which describes stimuli that appear achromatic when seen in their respective contexts. What the achromatic adjustments do not establish are which luminance pairs along the two loci correspond to stimuli that appear identical. Thus the achromatic adjustments do not completely determine the parameters of the diagonal model. Rather, they determine these parameters up to an unknown scale factor (see Appendix A for details).

To evaluate how well the achromatic adjustments predict the asymmetric matches, we proceeded as follows. For each observer we used the achromatic adjustments to determine the diagonal model parameters up to the unknown scale factor. We then used numerical search to determine the value of the scale parameter that minimized the prediction error of the diagonal model to the asymmetric matching data. The error was computed as $\Delta \mathrm{E} *$ in the CIELAB uniform col or space, just as when the diagonal model was fitted directly to the matching data.

The bottom panels of Fig. 1 show the quality of fit obtained for a subset of the asymmetric matching data from Observer ASH. The predictions obtained from achromatic loci (alternating fixation) are both close to the data and similar to those obtained from the full diagonal model (Fig. 1, top panels). As shown in Appendix A, the chromaticity of the predictions obtained from the achromatic loci are independent of the scale factor determined from the asymmetric matching data. Thus the chromaticity predictions may be obtained directly from the achromatic loci, without reference to asymmetric matches.

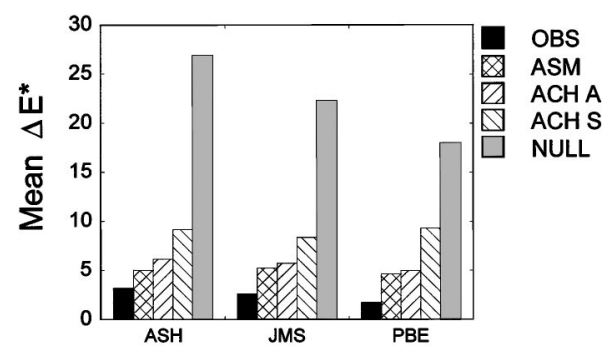

Fig. 3. Mean CIELAB $\Delta \mathrm{E} *$ prediction error for the asymmetric matching data set for each observer and each model. Abbreviations as in Table 1.

A summary comparison of the model fits is given in Fig. 3. The figure shows, for each observer and each model, the mean CIELAB $\triangle \mathrm{E} *$ prediction error. For comparison, two additional models are included in the summary. The observer model represents the variability in the data: The prediction for each match surface is the mean of all matches made for that surface. The null model repre sents the magnitude of the effect of context on appearance: Each asymmetric match is predicted by the test surface's tristimulus coordinates. The null model would provide good predictions if there were no effect of context.

The least-constrained fit is the full asymmetric matching model. This model results in a fit error closest to the observer error. Of the achromatic-constrained models, the fits obtained from the alternating-fixation data are better than those obtained from the steady-fixation data. The prediction errors for the alternating-fixation, achromatic-constrained model are similar to the errors for the full diagonal model. ${ }^{27}$ The prediction errors for the steady-fixation, achromatic-constrained model are noticeably greater than those for the alternating-fixation, achromatic-constrained model. This indicates that our summary comparison has discriminatory power: It is able to reveal the difference between the two fixation conditions.

\section{DISCUSSION}

Our results show that achromatic adjustments may be used to make accurate predictions of asymmetric matches. The predictions derived from a diagonal model constrained by the achromatic loci are almost as accurate as those derived from a diagonal model fitted-directly to the asymmetric matching data. This agreement is important. It suggests that when we study the effect of context on color appearance by measuring what looks gray, the results will also accurately characterize the effect of the same context change on the chromaticity of stimuli that appear colored.

Our condusion rests on the adequacy of the diagonal model for describing the asymmetric matching data. As may be seen in Fig. 3, the diagonal model does not describe our data set perfectly. Examination of the prediction residuals, however, did not reveal any systematic pattern of errors. If it is eventually possible to develop a model that provides a better description of the data, it re mains an open question as to whether achromatic adjustments will be able to determine the parameters of that 
model. It also remains open as to whether achromatic adjustments may be used to predict asymmetric matches under conditions where the diagonal model fails in a less subtle manner. ${ }^{23-25}$ Of particular interest to us are conditions used to study color constancy where both the illumination and the objects in the scene are varied. ${ }^{28} \mathrm{How}$ well the diagonal model performs for such conditions has not been extensively studied (but see Bauml ${ }^{20}$ ).

On the assumption that a diagonal model with the same parameters describes both achromatic adjustments and asymmetric matches, our conclusion follows from the derivation in Appendix A. The results presented here may be taken as support for this assumption. The fact that we can derive parameter values from the achromatic adjustments and use these to predict the asymmetric matches indicates that the two types of measurement are indeed affected by context in a consistent manner. Note, however, that the agreement between the two tasks depended critically on specifying the achromatic adjustment task so as to mimic the fixation strategy most natural for asymmetric matching. Other task-dependent factors could in principle have resulted in prediction failures. In addition, our results show that the measurement precision of the achromatic adjustments is sufficient to support extrapolations to asymmetric matches through the diagonal model.

In our alternating-fixation conditions, we had observers mimic the fixation strategy that we believe they would use in an asymmetric matching experiment. This manipulation had a measurable effect. The data in the top and bottom panels of Fig. 2 differ systematically. The fit error to the asymmetric matching data was higher for the model constrained by achromatic loci from the steadyfixation condition than for the model constrained by loci from the alternating condition. We can quantify the difference between the two fixation conditions by computing a constancy index from each set of achromatic loci, as described in Ref. 9. For comparison, we also computed a chromatic constancy index from the full diagonal model fitted to the asymmetric matching data. The constancy indices for each observer are given in Table 3. The mean index for the alternating-fixation condition is $70 \%$, similar to that for the asymmetric matching data (62\%). The constancy index for the steady-fixation condition is larger, $94 \%$. The latter difference is comparable to the difference between simultaneous color constancy measured with asymmetric matching and successive col or constancy measured with achromatic adjustment and steady fixation (compare Refs. 9 and 17). Thus much of the difference between simultaneous and successive color con-

Table 3. Constancy Indices ${ }^{a}$

\begin{tabular}{cccc}
\hline Observer & ASM & ACH A & ACH S \\
\hline ASH & 0.64 & 0.74 & 1.03 \\
J MS & 0.67 & 0.72 & 0.99 \\
PBE & 0.55 & 0.63 & 0.81 \\
\hline
\end{tabular}

a The constancy indices for the asymmetric matching experiment differ slightly from the chromatic constancy indices reported for the same data in Ref. 17 because here they are computed from the diagonal model rather than from the equivalent illuminant model. stancy may result from differences in the fixation strategies that are most natural for the two types of judgment.

\section{APPENDIX A}

In this appendix we describe how we used achromatic loci measured in two contexts to predict asymmetric matches across the same two contexts.

We represent stimuli by their cone coordinates, expressed as a column vector, e.g., $\mathbf{r}=\left(r_{1} r_{m} r_{s}\right)^{\top}$ where the $\mathrm{L}-, \mathrm{M}$ - and $\mathrm{S}$-cone responses $\mathrm{r}_{\mathrm{l}}, \mathrm{r}_{\mathrm{m}}$, and $\mathrm{r}_{\mathrm{s}}$ are computed with respect to the Smith-Pokorny estimates of the cone spectral sensitivities. ${ }^{29,30}$

The diagonal model for asymmetric matching states that a set of asymmetric matches is described by the equation

$$
\mathbf{r}_{\mathrm{T}}=\mathbf{D} \mathbf{r}_{\mathrm{M}}
$$

where $\mathbf{r}_{\mathrm{T}}$ represents the test, $\mathbf{r}_{\mathrm{M}}$ represents the match, and $\mathbf{D}$ is a $3 \times 3$ diagonal matrix that depends on the test and match contexts but is independent of $\mathbf{r}_{\mathrm{T}}$ and $\mathbf{r}_{\mathrm{M}} \cdot{ }^{15,17}$

Each point on a linear achromatic locus may be specified by a vector a of cone coordinates with unit length. The vector $\mathbf{a}$ is chosen so that it represents a light with the same chromaticity as the points on the achromatic locus. Each point on the locus has cone coordinates ca for some constant c. Let $\mathbf{a}_{\mathrm{T}}$ represent the achromatic locus measured under the context corresponding to the test stimulus in the asymmetric matching experiment, and similarly let $\mathbf{a}_{M}$ represent the achromatic locus measured under the context corresponding to the match. To link the achromatic loci with asymmetric matches, consider the stimulus $\mathbf{a}_{\mathrm{T}}$. This point appears achromatic when seen in the test context and should be an appearance match to some point on the achromatic locus $\mathbf{a}_{M}$ when that point is seen in the match context. Then for some constant c, we predict from the achromatic loci that

$$
\mathbf{a}_{\mathrm{T}}=\mathbf{D}\left(\mathrm{c} \mathbf{a}_{\mathrm{M}}\right)=\mathrm{cD} \mathbf{a}_{\mathrm{M}},
$$

where $\mathbf{D}$ is the diagonal matrix that describes asymmetric matches across the two contexts. This establishes that

$$
\mathbf{D}=\operatorname{diag}\left(\mathbf{a}_{\mathrm{T}} \cdot / \mathbf{a}_{\mathrm{M}}\right) / \mathrm{C},
$$

where the symbol ./ indicates entry-by-entry vector division and the function diag() creates a diagonal matrix with its argument on the diagonal. Thus the achromatic loci determine the matrix $\mathbf{D}$ up to an unknown scale factor $1 / \mathrm{c}$. When we used the achromatic loci to predict the asymmetric matching data, we computed D' $=\operatorname{diag}\left(\mathbf{a}_{\mathrm{T}} \cdot / \mathbf{a}_{\mathrm{M}}\right)$ and then used numerical search to find the value for $c$ that led to the matrix $\mathbf{D}=\mathbf{D}^{\prime} / \mathrm{c}$ that provided the best fit.

When we predict asymmetric matches with the diagonal model, note that two diagonal matrices $\mathbf{D}$ and $\mathbf{D}^{\prime}$ that differ only by a scale factor $\left(\mathbf{D}^{\prime}=\mathbf{C D}\right)$ yield predictions that differ only by the same factor. That is, for any test surface $\mathbf{r}_{\mathrm{T}}$ we have $\mathbf{D}^{\prime} \mathbf{r}_{\mathrm{T}}=\mathrm{cDr}_{\mathrm{T}}$. Since two predicted match surface coordinates that differ only by a scale factor have the same chromaticity, we may use the matrix $\mathbf{D}^{\prime}=\operatorname{diag}\left(\mathbf{a}_{\mathrm{T}} \cdot / \mathbf{a}_{\mathrm{M}}\right)$ to obtain chromaticity predictions for 
the asymmetric match to any test surface. When all we wish to predict is chromaticity, it is not necessary to determine the scale factor $1 / c$.

\section{ACKNOWLEDGMENTS}

This research was conducted as part of the first author's Ph.D. dissertation project. We thank J. D. Tietz for technical support and P. B. Egan and A. S. Haque for careful observing. Supported by National Institutes of Health Contract EY 10016.

*Correspondence should be sent to J. Speigle at his present address: Sharp Laboratories of America, 5750 NW Pacific Rim Boulevard, Camas, Washington 98607; e-mail, speigle@sharplabs.com.

\section{REFERENCES AND NOTES}

1. H. Helson and W. C. Michels, "The effect of chromatic adaptation on achromaticity," J . Opt. Soc. Am. 38, 1025-1032 (1948).

2. R. W. G. Hunt and L. M. Winter, "Color adaptation in picture-viewing situations," J. Phot. Sci. 23, 112-115 (1975).

3. J . S. Werner and J . Walraven, "Effect of chromatic adaptation on the achromatic locus: the role of contrast, luminance and background color," Vision Res. 22, 929-944 (1982).

4. R. S. Berns and M. E. Gorzynski, "Simulating surface colors on CRT displays: the importance of cognitive clues," in Proceedings of the AIC Conference: Colour and Light (Association Internationale de la Couleur, 1991), pp. 21-24.

5. M. D. Fairchild and P. Lennie, "Chromatic adaptation to natural and incandescent illuminants," Vision Res. 32, 2077-2085 (1992).

6. K. H. Bauml, "Color appearance: effects of illuminant changes under different surface collections," J. Opt. Soc. Am. A 11, 531-542 (1994).

7. D. H. Brainard and K. Ishigami, "Factors influencing the appearance of CRT colors," in Proceedings of the IS\&T/ SID Color Imaging Conference: Color Science, Systems, and Applications (Society for I maging Science and Technology, Springfield, Va., 1995), pp. 62-66.

8. E. J . Chichilnisky and B. A. Wandell, "Seeing gray through the on and off pathways," Visual Neurosci. 13, 591-596 (1996).

9. D. H. Brainard, "Color constancy in the nearly natural image. 2. Achromatic loci," J . Opt. Soc. Am. A 15, 307-325 (1998).

10. H. Helson, "Fundamental problems in color vision. I. The principle governing changes in hue, saturation and lightness of non-selective samples in chromatic illumination,"J . Exp. Psychol. 23, 439-476 (1938).

11. H. Helson and V. B. J effers, "Fundamental problems in color vision. II. Hue, lightness, and saturation of selective samples in chromatic illumination," J. Exp. Psychol. 26, 1-27 (1940).
12. J . J . McCann, S. P. McKee, and T. H. Taylor, "Quantitative studies in retinex theory: a comparison between theoretical predictions and observer responses to the 'Color Mondrian' experiments," Vision Res. 16, 445-458 (1976).

13. L. E. Arend and A. Reeves, "Simultaneous col or constancy," J. Opt. Soc. Am. A 3, 1743-1751 (1986).

14. A. Valberg and B. Lange-Malecki, "Mondrian complexity does not improve 'color constancy'," Invest. Ophthalmol. Visual Sci. Suppl. 28, 92 (1987).

15. D. H. Brainard and B. A. Wandell, "Asymmetric colormatching: how color appearance depends on the illuminant," J . Opt. Soc. Am. A 9, 1433-1448 (1992).

16. M. P. Lucassen and J. Walraven, "Color constancy under natural and artificial illumination," Vision Res. 36, 26992711 (1996).

17. D. H. Brainard, W. A. Brunt, and J . M. Speigle, "Color constancy in the nearly natural image. 1. Asymmetric matches," J . Opt. Soc. Am. A 14, 2091-2110 (1997).

18. W. S. Stiles, "Mechanism concepts in colour theory," J . Colour Group 11, 106-123 (1967).

19. J. M. Speigle and D. H. Brainard, "Luminosity thresholds: effects of test chromaticity and ambient illumination," J . Opt. Soc. Am. A 13, 436-451 (1996).

20. K. H. Bauml, "Illuminant changes under different surface collections: examining some principles of color appearance," J . Opt. Soc. Am. A 12, 261-271 (1995).

21. E. J . Chichilnisky and B. A. Wandell, "Photoreceptor sensitivity changes explain color appearance shifts induced by large uniform backgrounds in dichoptic matching," Vision Res. 35, 239-254 (1995).

22. J . von Kries, "Chromatic adaptation" [originally published in Festschrift der Albrecht-Ludwigs-Universitat (Fribourg, Germany, 1902), pp. 145-148.] In Sources of Color Vision, L. D. MacAdam, ed. (MIT Press, Cambridge, Mass., 1970), pp. 109-126.

23. D. J ameson and L. M. Hurvich, "Theory of brightness and color contrast in human vision," Vision Res. 4, 135-154 (1964).

24. J. Walraven, "Discounting the background: the missing link in the explanation of chromatic induction," Vision Res. 16, 289-295 (1976).

25. S. K. Shevell, "The dual role of chromatic backgrounds in color perception," Vision Res. 18, 1649-1661 (1978).

26. J. M. Speigle, "Testing whether a common representation explains the effects of viewing context on color appearance," Ph.D. dissertation (University of California, Santa Barbara, Calif., 1997)

27. A goodness-of-fit test does indicate that the difference between full diagonal and alternating-fixation, achromatic constrained models is statistically significant ( $F$-test for nested linear models applied to CIELAB E* errors; $p$ $<0.05$ for all observers.) This test, however, does not reflect prediction error induced by error in measurement of the achromatic loci.

28. J . M. Kraft and D. H. Brainard, "Mechanisms of color constancy under nearly natural viewing," Proc. Natl. Acad. Sci. USA 96, 307-312 (1999).

29. V. Smith and J. Pokorny, "Spectral sensitivity of the foveal cone photopigments between 400 and $500 \mathrm{~nm}$," Vision Res. 15, 161-171 (1975).

30. P. DeMarco, J. Pokorny, and V. C. Smith, "Full-spectrum cone sensitivity functions for X-chromosome-linked anomalous trichromats," J . Opt. Soc. Am. A 9, 1465-1476 (1992). 